\title{
Dynamics of Medical Specialties
}

Catalin-Iulian Chiurciu ${ }^{1}$, Adrian Nedelciu ${ }^{2}$, Dan Radoiu ${ }^{3}$, Tudorel Stirbu ${ }^{4}$ and Ion Dina ${ }^{5 *}$

${ }^{1}$ Romanian College of Physicians, Romania

${ }^{2}$ Expert Consultant IA, National Center for Healthcare Training, Bucarest, Romania

${ }^{3}$ Vianet Telecom Srl, Romania

${ }^{4}$ Databases Administrator, Bucharest College of Physicians, Romania

${ }^{5}$ Department of Gastroenterology and Internal Medicine Physician, Saint John Emergency Hospital, Romania

\begin{abstract}
The name of a medical specialty is a dynamic one because it changes according to chronological contextual needs. We analyzed 12 medical specialties nomenclatures for a 20 years period and we described the conceptual model of this change. The division, correspondence, renaming, fusion, creation, return and cancellation have been identified as operations, composing an "informational engine". We found 754 links between 692 different medical specialties instances. The resulting application is an electronic dictionary for the medical specialties correspondences. The project should be extended to all the European countries, even to create an online database for the next European professional card.
\end{abstract}

Keywords: Medical Specialties; Correspondences; Conceptual model; Nomenclature

\section{Dynamics of Medical Specialties}

\section{Background}

How many specialists in Pediatric Neurology do you have in your organization?

Excuse me, sir! You are interested in "Pediatric neurology" (the actual name of the specialty), "Infantile neurology" (the older name) or both? Do you agree to include the "Pediatric Neuropsychiatry" from 1993? Furthermore, please specify if you want the specialty name as on their original diplomas, the actual correspondences or as in the last certificate from the National College of Physicians?(An imaginary dialogue based on daily problems to query our database).

Dividing the entire contents of medical practice in formal entity in terms of limits of competence, with specific training curriculum and appropriate institutional opinion, depends on the frequency spectrum of current diagnostic procedures (clinical examination, imaging or laboratory) or therapeutic (medication, surgical procedures, other technical features). Variations occur both geographically (in terms of specific pathology and level of development of each country) and chronologically, the nomenclature of specialties requiring regular updates.

New course recommended by Medical Specialists European Union (EUMS) since 1993 is that they must regroup specialties on a common core training, including issues management, communication, Economy and Finance, together with the general clinical and professional basis. We can underline specialties which have suffered very few modifications as We highlight the specialties have changed very little as Ophthalmology, Obstetrics and Gynecology, Pediatrics and General Surgery. These last two specialties, even if they appear to be the same, have developed a variety of specialties derived (subspecialties). International medical associations, as EUMS (1), recommended that the specialties number should be kept under control, blocking creation of new specialties by medical or surgical approaches of a small area of pathology (Gastroenterology and Digestive surgery, for example). In the meantime we highlight the preservation of Neonatology as a distinct specialty in Romania, this one being a subspecialty of Pediatrics in all the other countries according with
Directive 2005/36/EC (2). Also the specialty of Diabetes, metabolic and nutritional diseases is associated with Endocrinology in most of the other member States. Other specialties, as Functional Explorations, were cancelled by division because their area became extremely large, impossible to be covered by one specialist. In this case each specialty has included the specific exploratory field. There are also specialties which have generated, in time, by strong needs, new specialties, due to the same explanation as in case of Functional explorations. Internal medicine remained a distinct specialty but has now a lot new relatives as Rheumatology, Pneumology, Hematology, Gastroenterology, Geriatrics, with much narrow areas of practice. This approach creates the opportunity to see how in a moment in time, there are doctors who practice the same specialty under different names, depending on when they have become specialists. We believe that the very rapid development of knowledge and medical practice itself will support a permanent process of adaptation to surrounding realities due to the dynamics of the pathology, practice approach, postgraduate specialized medical training and, the last but not least, the influence of political environment and interest groups [1,2].

\section{Defining the Problem}

\section{Motto: Solving a problem correctly depends on our ability to fully define it}

In all formal aspects of the clinical practice, but especially in providing statistic reports for the medical management, we face the problem of giving the actual denomination to a specialty, used in the official updated List of Specialties (LOS), instead of the specialty denomination (SD), obtained years ago. Regarding the medical practice, the correspondences allow the precise classification of the professional

*Corresponding author: Ion Dina, Department of Gastroenterology and Internal Medicine Physician, Saint John Emergency Hospital, Romania, Tel: 02133451 90; E-mail: endo_din@yahoo.com

Received October 09, 2015; Accepted December 11, 2015; Published December 18,2015

Citation: Chiurciu Cl, Nedelciu A, Radoiu D, Stirbu T, Dina I (2015) Dynamics of Medical Specialties. J Biom Biostat 6: 267. doi:10.4172/2155-6180.1000267

Copyright: (c) 2015 Chiurciu $\mathrm{Cl}$, et al. This is an open-access article distributed under the terms of the Creative Commons Attribution License, which permits unrestricted use, distribution, and reproduction in any medium, provided the original author and source are credited. 
standard competencies of a physician, who is certified according to a cancelled list of specialties, but practicing according to the updated list. This shows that specialties, above their abstract appearance, are the doctors who practice in person. For statistic reports, based on a long period of time, the use of specialty name as aggregation criteria could be inconsistent, if there are more entities attributed to the same specialty. As you can see, the correlation can and should become a sine qua non standard mechanism, crucial for a correct, appropriate certification. Today, without this mechanism, we have errors in our work:

The lists of specialties published by different operators are frequently in disharmony with the legal updated LOS,

The lists of medical specialties include frequently different denominations for the same medical specialty

None of the operators offers at least the previous denomination of a medical specialty

The manual adjustment of the denomination update might cause erroneous results

We believe that the examples and the theory emphasizes the real scale of the problem, providing arguments for the need to implement an online engine for dynamic management of specialties, as a reference for all operators that use the medical information system.

\section{Goal}

The purpose of this presentation is to create a standard conceptual model for a search engine as support for the management of the medical specialties.

What we propose is a 'must have' for the competent institutions (College of Physicians, Ministry of Health, Health Insurance Authority) as an operational support for the official papers elaboration.

\section{Methods}

We developed a theoretical framework based on hypotheses that characterize a dynamic relationship between the denominations of medical specialties in different periods of time.

Each of these suppositions was validated regarding the capacity of defining a conceptual pattern and the integration in the functional pattern of the legal arrangements in the last 20 years.

\section{Conceptual model}

Entities and relations: We operate with three categories of components: Orders of the Ministry of Health (OMH): Documents defined by a number and a date when it is operated [3-14]. Each OMH introduces a LOS a LOS

Specialties: component names corresponding to individual within

Links: Correspondence between two specialties, strictly defined by the involved specialties

Theoretically, each primary connection can participate in many types of relations, according to the reference point (Figure 1). Thus, S1 and $\mathrm{S} 3$ are connected by elementary link $\mathrm{L} 1$, and $\mathrm{S} 2$ and $\mathrm{S} 3$ are connected by L2 elementary link. Together, however, the links L1 and L2 define a fusion (the complex relationship R1) and the links L4 and L5 define a division (R3 complex relationship). Specialty S3 is linked upstream (with its correspondents from previous $\mathrm{OMH}$ ) by the relation R1, and downstream (with its correspondent for the next $\mathrm{OMH}$ ) by the relation

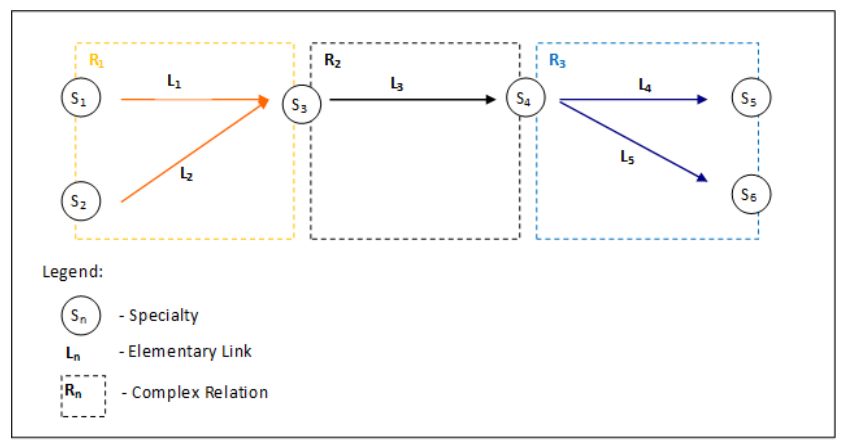

Figure 1: Definingentities and relations.

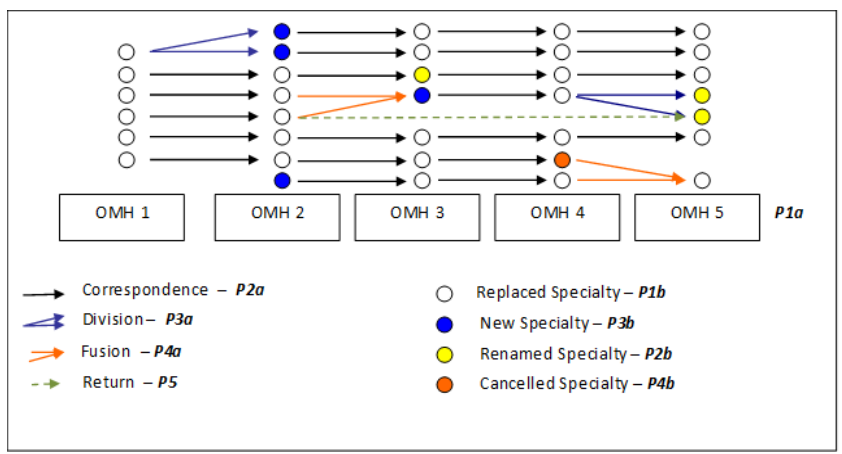

Figure 2: Conceptual model and hypotheses.

R2. Similarly, Specialty S4 is linked upstream (with its correspondent from previous $\mathrm{OMH}$ ) by the relation $\mathrm{R} 2$, and downstream (with its correspondent for the next $\mathrm{OMH}$ ) by the relation $\mathrm{R} 3$.

Hypotheses: The first issue that influenced the definition of conceptual model was crucial if the nomination of the same Specialty in different $\mathrm{OMH}$ represents instances of the same entity or even the entity itself, which will retain its identity, changed over time. Analyzing the behavior of the Specialties for each of the relations established between them have chosen the initial hypothesis of this change of identity for each new $\mathrm{OMH}$, even if the name remains unchanged. We considered that this is the most general concepts and preservation of identity can be regarded as a somewhat particular case (Figure 2).

P1a: Each OMH introduces new Specialties entities, even if some of them kept the same names.

The second problem, derived from the first, is whether an $\mathrm{OMH}$ mentioning only the changes to the above must be regarded as an entire LOS or just a variation of the previous LOS. To maintain the generality, we chose the explicit expansion of all LOS even for the OMH which publish only the changes from the previously $\mathrm{OMH}$.

P1b: Each OMH reintroduces the whole LOS as separate entities.

The third issue is the types of relations can be established between specialties. Apparently, a medical specialty may have a route such as: creation (if not previously existed), validity (the period is effective) and cancellation (at the time the content that defines it no longer corresponds to reality or contextual needs). In reality:

- In accordance with the hypotheses P1 and P2, any new OMH changing the relevant entities corresponding to any Specialty, even 
though it retains the name. Therefore, the validity is a successive replacement of different entities with the same name. There is the situation in which an $\mathrm{OMH}$ changes the previous name of a Specialty, in which case we talk about renaming, as a particular case of replacement.

P2a: Replacement is 1:1 correspondence between two specialties from successive $\mathrm{OMH}$.

P2b: When the name of a Specialty changes, it is a particular case, Renaming.

The establishment of a new Specialty means often the splitting of an already existing specialty (see the previous example regarding the Internal medicine), due to the particular development of its composing areas, which we call partition. This splitting consists also in the dividing of the physicians, confirmed before in the parent specialty, in respect of their main preoccupation in the field. The parent Specialty may be preserved as such or it may be cancelled (the specialty of Pediatric Neurology and Psychiatry was divided in Pediatric Neurology and Pediatric Psychiatry and the previous Specialty was cancelled).

P3a: Division is the $1: n$ correspondences between several specialties of successive $\mathrm{OMH}$.

P3b: Division can be done with or without taking the initial name of the child Specialty.

Whereas Specialty is related to those doctors who practice it, the cancellation of a Specialty is not a real phenomenon, because it would mean that those physicians should be removed abruptly from their work. In reality, these doctors are allowed to continue pursuing his profession in a Specialty of fusion, so that cancellation is often a fusion of two previous Specialties while keeping the name of one of the Specialties prior (Orthopedics and Traumatology fusion with Pediatric Orthopedics results Orthopedics and Traumatology). There are situation where the field of activity of two different Specialties come to overlap significantly, taking place in this case a fusion under a new, common denomination (Allergology and Clinical Immunology, Anesthesia and Intensive Care).

P4a: Fusion is the correspondence n: 1 between two or more Specialties from successive OMH.

P4b: Fusion can be done with or without taking the initial name of one of the Specialties.

In some cases, fusion or division of Specialty proved inoperative or unimplemented, for which those operations must be restored fully or partly by a return to a situation similar to the previous one. In this case establishes a relationship of return, as the case of correspondence between specialties but remote in time.

P5: Correspondence between specialties is between successive $\mathrm{OMH}$. Return is the correlation between the specialties of remote $\mathrm{OMH}$.

\section{The Physical Model}

\section{Basic data - OMH and specialties}

For the study period, $01.01 .1990-31.12 .2009$, there were identified 12 LOS, successively introduced by law texts according to $\mathrm{OMH}$ (Table 1). Totaling the number of specialties introduced by all $12 \mathrm{MOH}$ there were identified a total of 692 entities, operated in the period under review (3-14).

Each of the OMH either fully resumes all LOS coming into force

\begin{tabular}{|c|c|c|}
\hline No & OMH date & OMH number \\
\hline 1 & 30.05 .1990 & OMH 538/1990 \\
\hline 2 & 11.08 .1993 & OMH 990/1993 \\
\hline 3 & 22.05 .1995 & OMH 954/1995 \\
\hline 4 & 04.06 .1999 & OMH 378/1999 \\
\hline 5 & 12.11 .1999 & OMH 772/1999 \\
\hline 6 & 04.04 .2000 & OMH 254/2000 \\
\hline 7 & 18.12 .2001 & OMH 923/2001 \\
\hline 8 & 09.03 .2004 & OMH 197/2004 \\
\hline 9 & 24.09 .2004 & OMH 1138/2004 \\
\hline 10 & 31.08 .2006 & OMH 1044/2006 \\
\hline 11 & 17.07 .2007 & OMH 1142/2007 \\
\hline 12 & 11.09 .2008 & OMH 1508/2008 \\
\hline & Table 1: The 12 OMH in studied period. \\
\hline
\end{tabular}

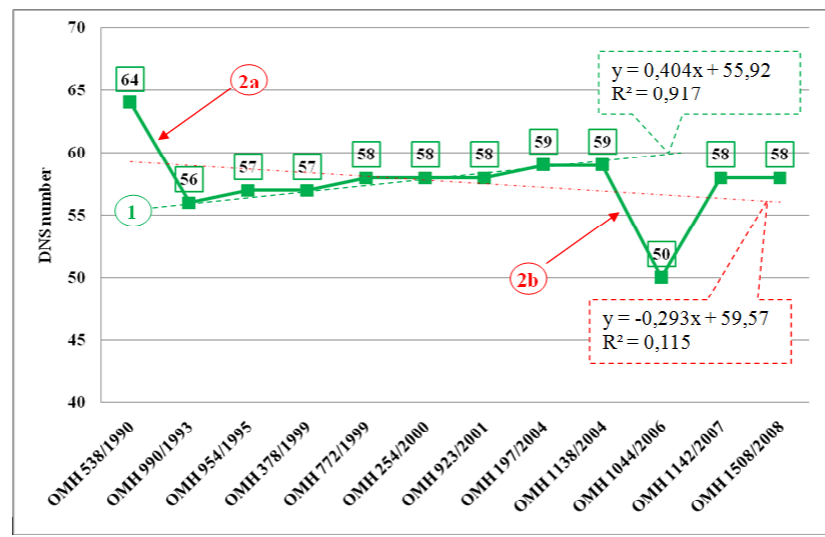

Figure 3: The Dynamic Number of Specialties (DNS) in successive LOS.

beginning with the publication day or strictly states the amendments to the OMH, the full LOS being reconstituted by default. The number of specialties introduced by an OMH varies between 64 (first $\mathrm{OMH}$ of the study, 538/1990) and 50 (OMH 1044/2006) - argument for hypothesis P1a). Two classes of phenomena are observed (Figure 3):

1. The net growing trend in the number of specialties. Thus, between $1993-2004$, associate trend line series is defined by a positive factor for 0.404 (almost a new specialty every $2 \mathrm{OMH}$ ), with a very good correlation coefficient $(\mathrm{R} 2=0.917)$.

2. Periodically drastic reductions appear in the number of specialties.

in 1993: reducing by 8 of the total number of specialties

in 2006: reducing by 9 of the total number of specialties

These periodic reductions in the number of specialties are followed by natural "rebound's" and further increases but not to previous levels, managing to give the series a negative trend coefficient $(-0.293)$. The "artificial" appearance of the interventions is still highlighted by the weak coefficient of correlation obtained for the global trend line (R2 $=0.115)$.

\section{Relations between specialties}

For each of the names corresponding to an $\mathrm{OMH}$ there have been set matches with the corresponding names from the previous $\mathrm{OMH}$ or OMHs, as stated in the text of that law. Among the 692 specialties there were identified 732 primary links, of which 710 links between 
specialties from successive $\mathrm{OMH}$ (primary correspondents) and 22 links between specialties in remote $\mathrm{OMH}$ (jumps). It may be noted that the jumps occur whenever the sharply reduction of the number of specialties is followed by a return to the previous number of Specialties and are meant to restore previously fusion correspondences between Specialties and their counterparts after re dividing (Figure 4).

All primary connections underline complex operations previously described in the conceptual model. Thus, for each of the specialties mentioned in the OMHs, primary links with corresponding specialties from the $\mathrm{OMH}$ were successively integrated into the complex links (relations), resulting in the following range:

1) Downstream relations: All 692 specialties related to their immediate counterparts in downstream through 634 Relations (argument for hypothesis P1b):

\section{a) 625 1:1 correspondence from which:}

i) 554 correspondences between entities of the same name Replacements (argument hypothesis P2a).

ii) Correspondence between 71 entities with different names Renaming (argument hypothesis P2b).

b) 9 correspondences $1: \mathbf{n}$ - division (argument hypothesis P3a).

The last 58 specialties, corresponding to the present $\mathrm{OMH}$, not related to other specialties by downstream relations.

2) Upstream relations: Each of the 692 specialties are related to their counterparts in 684 upstream relations (hypothesis argument P1b):

\section{a) 663 1:1 correspondences from which:}

i) 611 correspondences between entities of the same name Replacement (argument hypothesis P2a).

ii) 52 matches between entities with different names - Renaming (argument hypothesis P2b).

\section{b) 21 matches n: 1 - fusion (argument hypothesis P4a)}

Top 64 specialties, corresponding to the initial OMH and the 8 new specialties that appeared along the way are not related to other specialties by upstream relations.

It is noted that the number of substitutions described in the

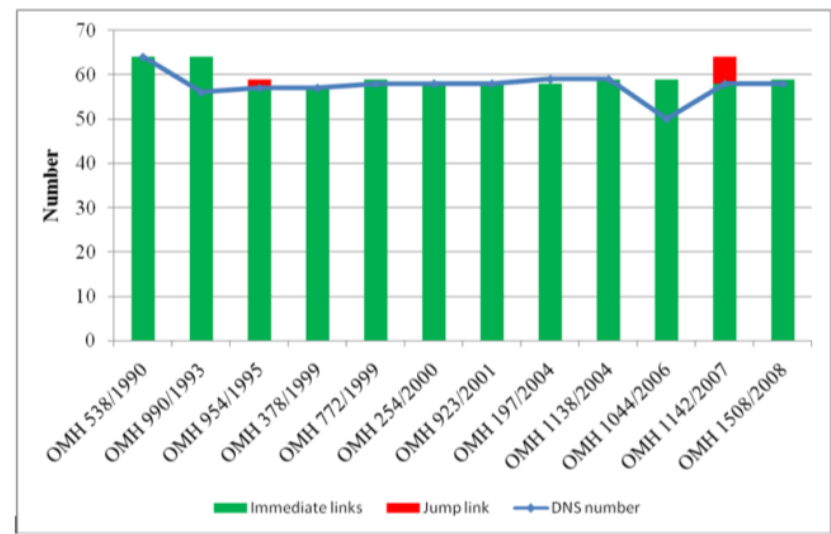

Figure 4: Thecorrelation between links number and DNS number in successive LOS. downstream relationship differs from that described in the upstream relationship. The explanation for this phenomenon is that:

When a specialty merges with more specialties belonging to a previous $\mathrm{OMH}$, while one of them keeps the name, for the specialty keeping the name the relation is described in upstream, while for the other specialties it is described the replacement relation. Obviously, the resulting Specialty will relate to an upstream division. Thus, a division described in the "downstream relationship" chapter will have as a correspondent a replacement and one or more renames in the "upstream chapter" (hypothesis argument $\mathrm{P} 3 \mathrm{~b}$ ).

Where several specialties in one $\mathrm{OMH}$ converge to a single Specialty in previous $\mathrm{OMH}$, maintaining the name of one of them, for the Specialty retaining the name describes the downstream relationship of replacement, while for other specialties it is described the Renaming relationship. Obviously, the resulting Specialty will have as upstream relationship, a Fusion. Thus, to a fusion described in the "upstream relationships" chapter have as correspondent a replacement and one or more renaming in the "downstream relationships" chapter (argument for hypothesis $\mathrm{P} 4 \mathrm{~b})$.

3) Jumps: Amongst the 692 specialties there were described 22 links between remote in time

OMHs, to define the return of some specialties divided in to their previous correspondents before fusion (argument hypothesis P5).

\section{Illustration of Specific Instances}

Functional model was developed under MS SQL Server 2005 Express, with an ASP application. Data were loaded into a database with three tables, one for OMH, the second for Specialties and the third for Links. Hypotheses were the basis for developing a function with two input parameters, The Reference Specialty and the date for which we search the correspondences, resulting in a corresponding table with Specialty or Specialties. Depending on the date position relative to the $\mathrm{OMH}$ introduction date, function returns correspondent Specialties upstream (for earlier introduction of specialty) or downstream (for the later introduction of specialty).

\section{Example 1a}

Fusion of Infantile Neurology with Infantile Psychiatry into Infantile Neurology and Psychiatry, with further Redividing into Pediatric Neurology and Pediatric Psychiatry

Input parameter: Infantile Neurology and Psychiatry, (corresponding to OMH from 990/1993).

Output:

- Pediatric Neurology

From: 05.06.1999

- Pediatric Psychiatry

- Pediatric Psychiatry

From: 23.05.1995

- Pediatric Neurology

From: 12.08.1993 - Infantile Neurology and Psychiatry

From: 31.05.1990 - Infantile Neurology

- Infantile Psychiatry 
It is noted that following the run function for previous OMH (1990) are found two matches (Infantile Neurology and Infantile Psychiatry), which shows that the Specialty of entry was the result of a Fusion. For the next OMH (1995), we obtain all two matches (Pediatric Neurology and Pediatric Psychiatry), corresponding to a division.

\section{Example 1b}

Fusion of Infantile Neurology with Infantile Psychiatry into Infantile Neurology and Psychiatry and only one correspondences of Infantile Neurology after the redividing, Pediatric Neurology

Input parameter Infantile Neurology, (corresponding to $\mathrm{OMH}$ from 538/1990

Output:

From: 23.05.1995 - Pediatric Neurology

From: 12.08 .1993 - Infantile Neurology and Psychiatry

From: 31.05 .1990 - Infantile Neurology

It is noted that running the function for the next $\mathrm{OMH}$ will get Pediatric Neurology and Psychiatry, but due to jump connection (return), for the second $\mathrm{OMH}$ is achieved only one correlation from the two specialties in which Infantile Pediatric Neurology and Psychiatry divided, Pediatric Neurology.

\section{Conclusions}

Functional model developed based on the conceptual model and loaded with real data from the period studied into an automated environment, which permit the emulation of all links between medical specialties operated during this period. The testing was done not only abstract, to the specialty as independent entities, but also by checking the path traveled by the doctors members of the Bucharest College of
Physicians. Engine management functionality validates the conceptual model described, and the degree to cover all the correspondences closely by loading the links between specialty shows that the conceptual model is not only fair but sufficient. This finding argues the possibility of extending this approach to defining specialties from around the country, opening the prospect of electronic correspondence with other European countries, even as a basis towards professional European card.

\section{Acknowledgment}

The work was supported by the Medical Foundation "St. Luke".

\section{References}

1. European Union of Medical Specialists (1993) Charter on training of medical specialists in the European Community.

2. (2008) European Parliament and Council-Directive 2005/36/EC on the recognition of professional qualifications (with 2008 amendments).

3. Romanian Ministry of Health - Order 538/1990 regarding Medical Specialties.

4. Romanian Ministry of Health - Ordinul 990/1993 regarding Medical Specialties.

5. Romanian Ministry of Health - Order 954/1995 regarding Medical Specialties.

6. Romanian Ministry of Health - Order 378/1999 regarding Medical Specialties.

7. Romanian Ministry of Health - Order 772/1999 regarding Medical Specialties.

8. Romanian Ministry of Health - Order 254/2000 regarding Medical Specialties.

9. Romanian Ministry of Health - Order 923/2001 regarding Medical Specialties.

10. Romanian Ministry of Health - Order 197/2004 regarding Medical Specialties.

11. Romanian Ministry of Health - Order 1138/2004 regarding Medical Specialties.

12. Romanian Ministry of Health - Order 1044/2006 regarding Medical Specialties

13. Romanian Ministry of Health - Order 1142/2007 regarding Medical Specialties. 14. Romanian Ministry of Health - Order 1508/2008 regarding Medical Specialties. 